\title{
Perioperative cerebral oxygen saturation complexity in predicting cardiac surgical outcomes
}

\author{
C.W. Wang ${ }^{1}$, T. D. Wu' ${ }^{2}$, T. Y. Lin ${ }^{1}$, C. W. Lu ${ }^{1}$ \\ ${ }^{1}$ Far Eastern Memorial Hospital - New Taipei City (Taiwan), ${ }^{2}$ National Taiwan University \\ Hospital - Taipei City (Taiwan)
}

\section{Background and Goal of Study}

By using brain as a vital organ, cerebral near-infrared spectroscopy (NIRS) is widely studied for its association with major organ morbidity and mortality (MOMM). The complexity of biosignals, such as heart rate or intracranial pressure, was applied to predict poor clinical outcomes. The cerebral NIRS signals complexity is proved to correlate with mortality in critically ill preterm infants. However, we had little information about the prognostic effect of cerebral oxygen saturation complexity. The aim of this study is to determine whether the complexity of the cerebral NIRS signals can better predict the clinical outcomes in cardiac surgery.

\section{Materials and Methods}

Total 80 patients scheduled for cardiac surgery were reviewed retrospectively. Cerebral NIRS monitor was applied routinely to measure perioperative cerebral oxygenation in cardiac surgical patients. We used multiscale entropy (MSE) analysis to evaluate the complexity of cerebral oximetry values. Baseline and mean values of intraoperative cerebral oxygenation were also recorded. Independent $t$ test, ROC analysis, and logistic regression were applied to exam the correlation to major organ morbidity and mortality.

Fig01a

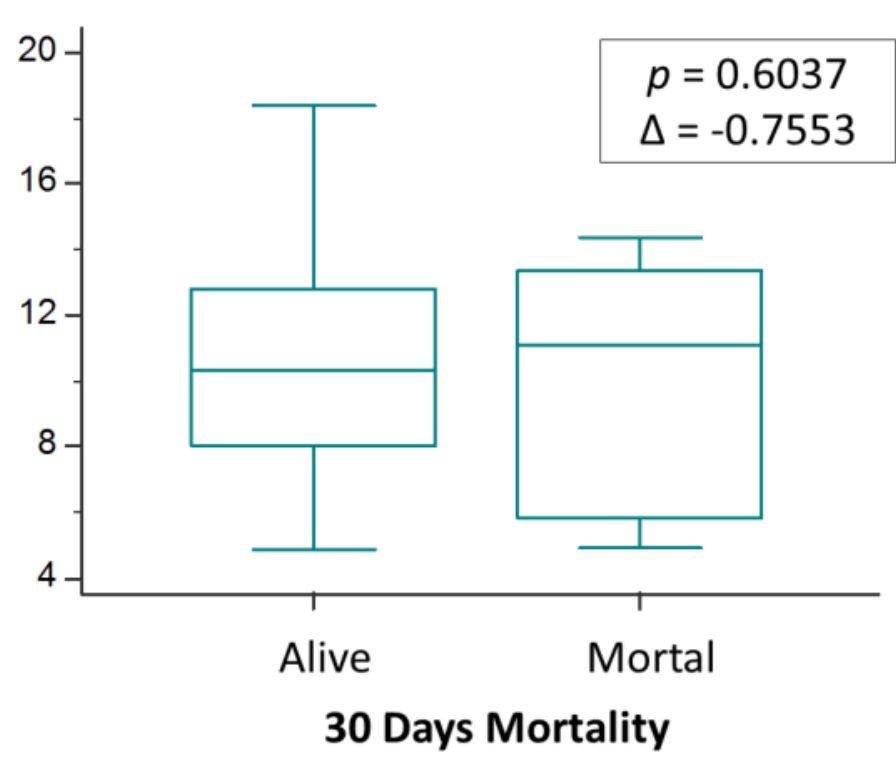

Fig01d

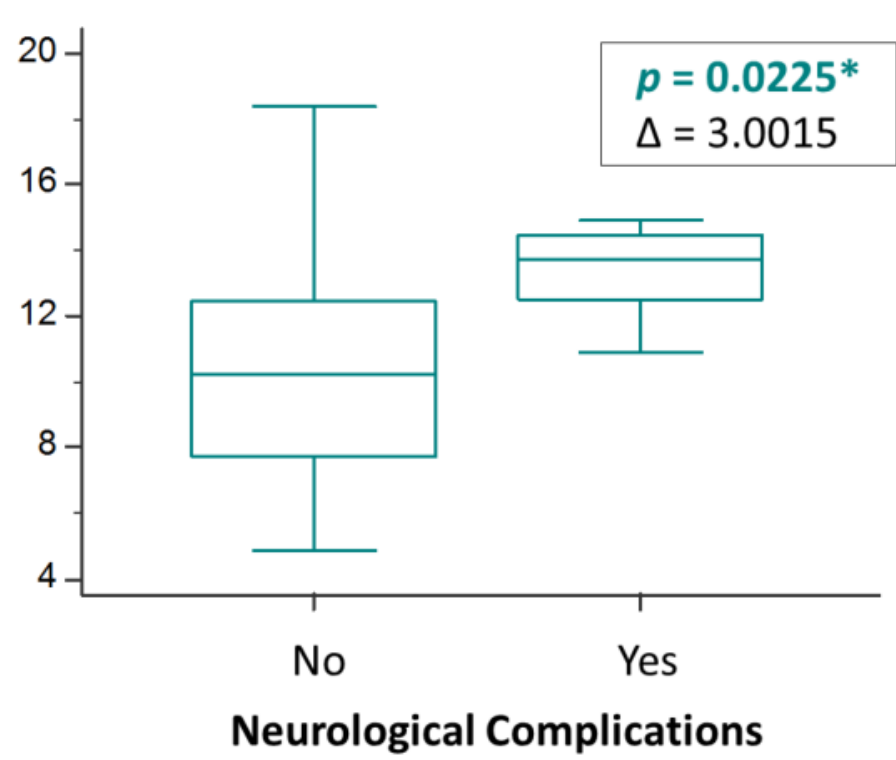

\section{Results}

Among major organ morbidity and mortality, the complexity of cerebral saturation was only correlated with adverse neurological outcomes. The patients with postoperative neurological complications had significant higher cerebral oximetry complexity $(p<0.001)$, lower perioperative mean oximetry value $(p=0.04)$, but had no difference in baseline cerebral saturation $(p=0.08)$. Besides, cerebral oximetry complexity was an independent predictor of adverse neurological outcomes $(p<0.001)$.

\section{Conclusion}

Complexity of peri-operaitve cerebral saturation can be applied to predict the clinical neurological outcomes better than baseline or average intraoperative cerebral oximetry values can in cardiac surgical patients.

Table 1. Factors predicting neurological outcomes: multivariate logistic regression analysis

\begin{tabular}{lrrl}
\hline \multicolumn{1}{c}{ Variables } & Coefficient & Std. Error & $p$ \\
\hline Cerebral oximetry complexity & 0.58142 & 0.22512 & $0.0098^{*}$ \\
Baseline cerebral oximetry value & -0.09206 & 0.06599 & 0.1630 \\
Average cerebral oximetry value & -0.06348 & 0.07534 & 0.3994 \\
\hline
\end{tabular}

$* p<0.05$
Fig01b

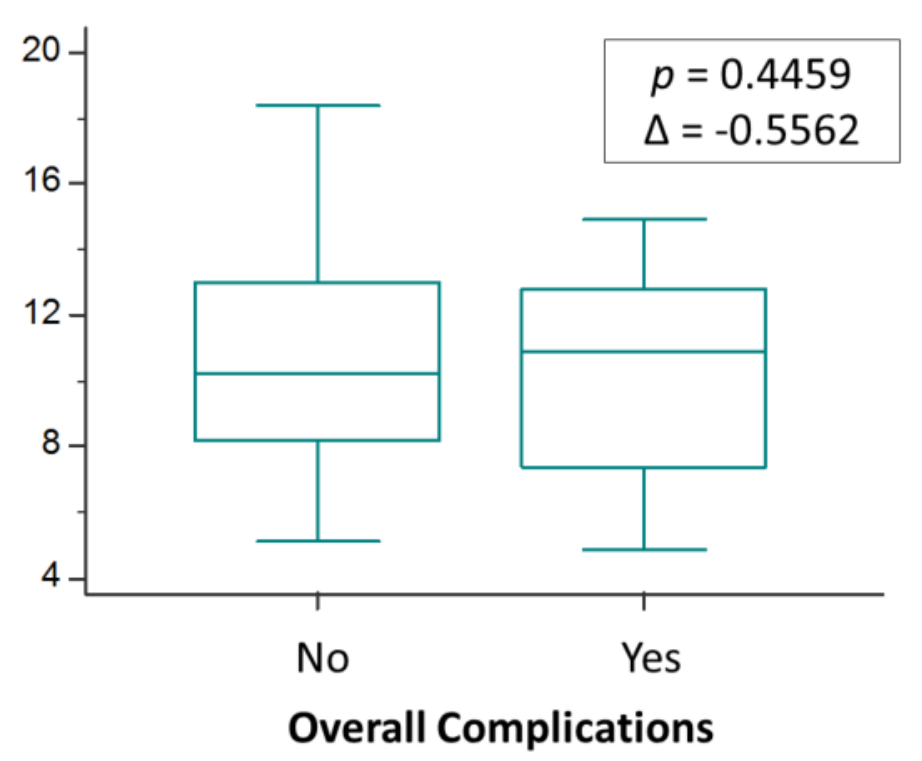

Fig01e

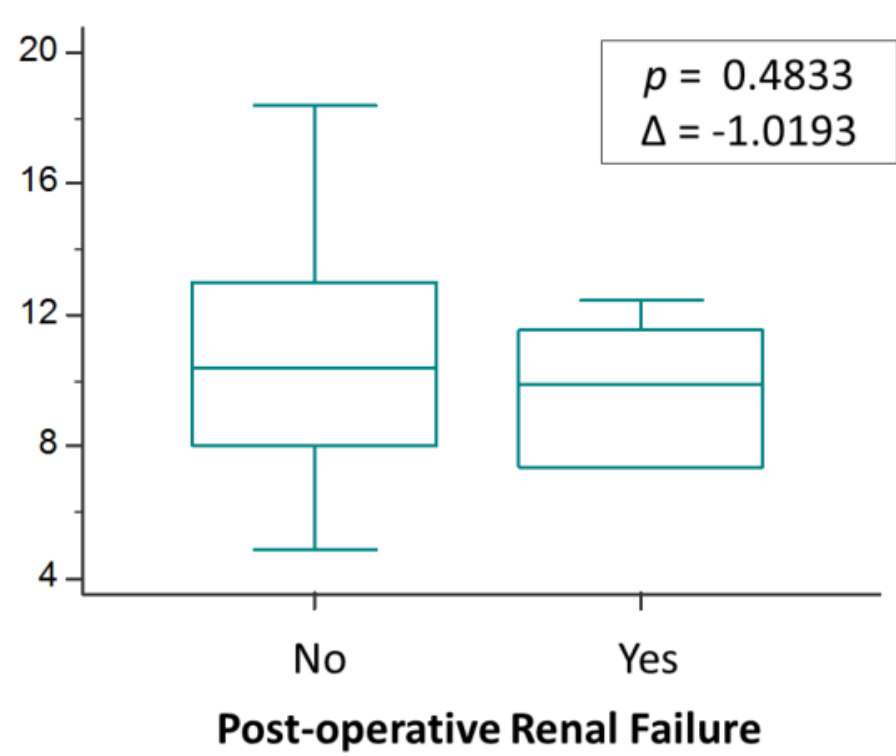

Fig01c

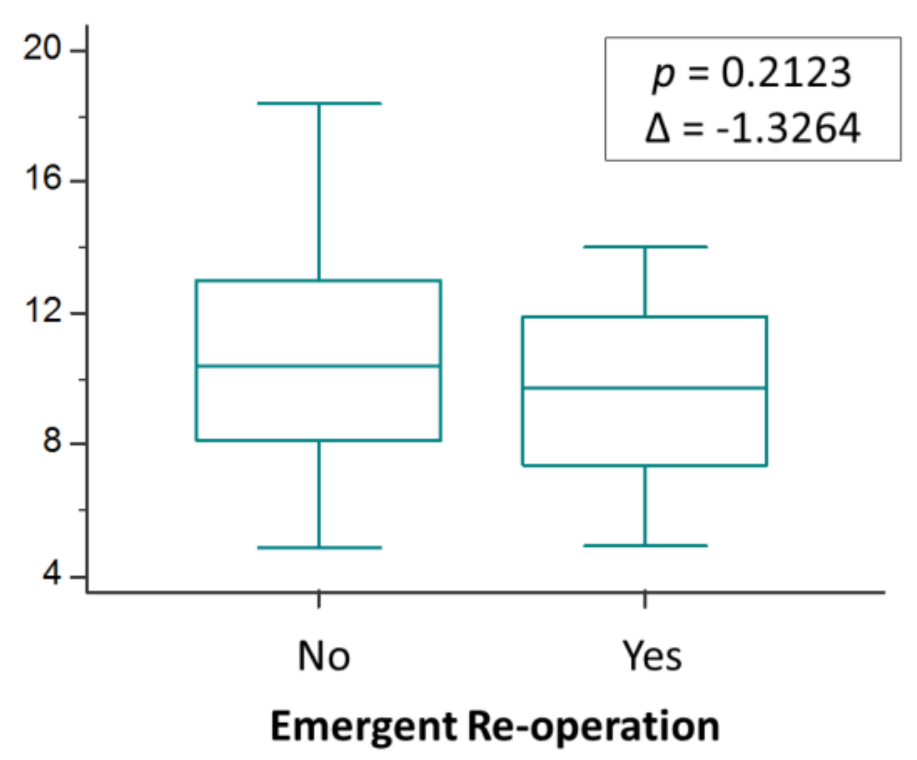

Fig01f

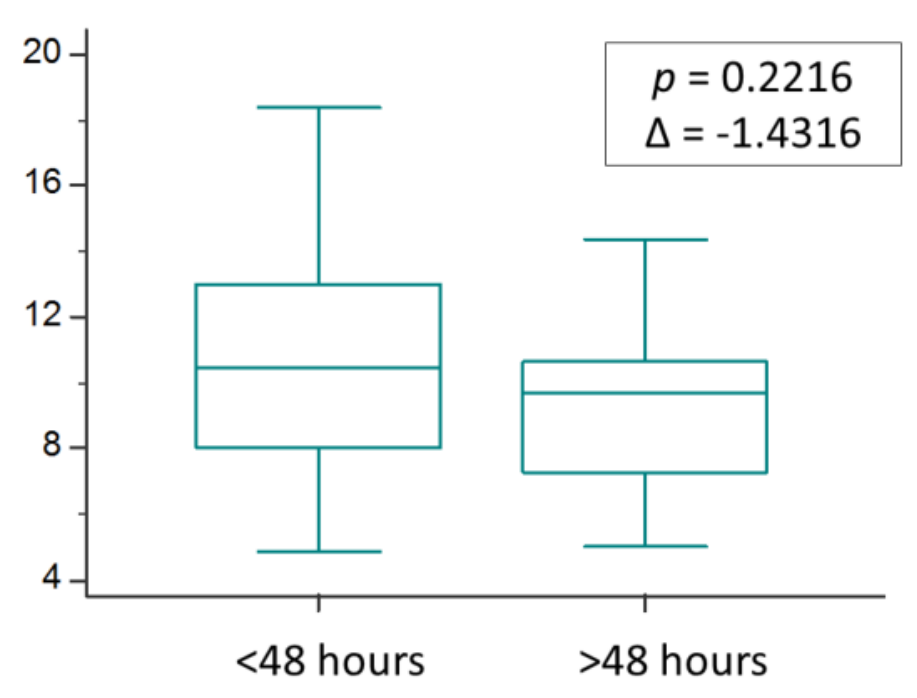

Post-operative Mechanical Ventilation

Figure01a-f. Independent t test of brain oximetry complexity on major organ morbidity and mortality. ${ }^{*} p<0.05$ 